\title{
Absolute Energy Levels of Liquid Water
}

\author{
Francesco Ambrosio, ${ }^{*}$ Zhendong Guo, and Alfredo Pasquarello \\ Chaire de Simulation à l'Echelle Atomique (CSEA), Ecole Polytechnique Fédérale de \\ Lausanne (EPFL), CH-1015 Lausanne, Switzerland \\ E-mail: Francesco.Ambrosio@epfl.ch \\ Phone: +4121 6933423. Fax: +41216935419
}

*To whom correspondence should be addressed 


\begin{abstract}
We determine the ionization potential (IP) and the electron affinity (EA) of liquid water together with the absolute redox level of the standard hydrogen electrode (SHE) by combining advanced electronic-structure calculations, ab initio molecular dynamics simulations, thermodynamic integration, and potential alignment at the water-vacuum interface. The calculated SHE level lies at $4.56 \mathrm{eV}$ below the vacuum level, close to the experimental reference of $4.44 \mathrm{eV}$ inferred by Trasatti. The band edges are determined through a hybrid functional designed to reproduce the band gap achieved with highly accurate $G W$ calculations. Our analysis yields $\mathrm{IP}=9.7 \mathrm{eV}$ and $\mathrm{EA}=0.8 \mathrm{eV}$, consistent with both photoemission spectra of liquid water and thermodynamical data for the hydrated electron.
\end{abstract}

Liquid water is ubiquitous in science, due to its apical role in a variety of relevant physical, chemical, and biological processes. ${ }^{1-3}$ However, despite the gargantuan number of both experimental and theoretical studies devoted to liquid water, the scientific community has not yet reached a consensus on its electronic structure. ${ }^{4-8}$ In particular, three levels are of particular relevance, viz. the valence band edge, the conduction band edge, and the redox level pertaining to the standard hydrogen electrode (SHE). The valence band edge defines the ionization potential (IP) and has been measured through photoemission spectroscopy of liquid water and $\mathrm{NaOH}$ aqueous solutions but available data fall within a wide interval ranging from 9.3 to $10.1 \mathrm{eV}$ below the vacuum level. ${ }^{9-12}$ In this context, it should be noted that current techniques are capable of accurately probing the electronic structure of the bulk, ${ }^{12}$ and hence the large indetermination in the measured values should not been assigned to the sensitivity to the electronic structure at the surface. Moreover, the variation of the ionization potentials in the vicinity of the surface has been found to be negligible from theory. ${ }^{8}$ The conduction band edge, which defines the electron affinity (EA), or equivalently its position vs. the vacuum level (denoted as $V_{0}$ ), has remained even more elusive, as no direct measurement of this level has so far been possible. Hence, available estimates rely on various modelling 
schemes ${ }^{4,5,7}$ mainly feeded by thermodynamical data for the hydrated electron, ${ }^{13,14}$ and have only recently been analyzed in a critically way to yield $V_{0}=-0.97 \mathrm{eV} .^{7}$ In this context, the SHE level carries particular relevance as it represents the internal reference of choice, when energy levels in aqueous solution or at water-semiconductor interfaces are addressed. ${ }^{15-18}$ The computational SHE based on the reduction of an aqueous hydronium cation to gaseous hydrogen has been instrumental to identify this level with respect to the band edges of liquid water. ${ }^{15,19}$ However, its alignment with respect to the vacuum level has remained poorly investigated in theoretical studies, despite the availability of a reliable experimental reference at $-4.44 \mathrm{eV}$ due to Trasatti. ${ }^{20}$ Hence, a theoretical description that reconciles the available experimental data is particularly timely.

Electronic-structure calculations have struggled in providing a well-defined picture of the energy levels. Standard hybrid functionals and one-shot $G_{0} W_{0}$ calculations yield band gaps varying between 6.2 and $7.3 \mathrm{eV}{ }^{6,15,19,21,22}$ severely underestimating plausible experimental values, which are expected to range between 8.3 and 9.1 on the basis of the band edges given above. $^{7,9-12}$ One complication is that nuclear quantum effects (NQEs) cannot be neglected as they lead to a reduction of the band gap by $0.5-0.7 \mathrm{eV} \cdot{ }^{6,8,23}$ Accounting for NQEs, quasiparticle selfconsistent $G W(\mathrm{QS} G W)$ (Ref. 6) and $G_{0} W_{0}$ on top of range separated hybrid functionals (Ref. 8) agree in yielding band gaps in the range of $9.8-10.05 \mathrm{eV}$. However, it is well documented that vertex interactions should be accounted for to achieve highly accurate band gaps for a wide range of materials. ${ }^{24-27}$ This has been achieved for liquid water with the QSG $\tilde{W}$ calculations by Chen et al., which result in a band gap of $8.9 \mathrm{eV}$ and are supported by the excellent agreement with photoemission spectra of the occupied states. ${ }^{6}$

The determination of the band edge levels is subject to more uncertainty. The QSGE calculations estimate the IP and EA at 9.4 and $0.5 \mathrm{eV}$, respectively, but the alignment to the vacuum level relies on the experimental SHE level. ${ }^{6}$ Alternatively, Gaiduk et al. found respective values of 10.25 and $0.2 \mathrm{eV}$ accounting for the calculated potential offset at the water-vacuum interface. ${ }^{8}$ However, the latter estimate for the EA conflicts with the value of 
$V_{0}$ inferred from the hydrated electron $(-0.97 \mathrm{eV}$, Ref. 7$)$ and might be flawed by the large band gap resulting from the neglect of vertex corrections. ${ }^{6,28}$ Using the same configurations obtained in Ref. 6 and the alignment in Ref. 8 , a recent $G W_{0}$ study, with the self-consistency being only partially carried out for the band energies, determines a yet different set of values for the IP and EA, at 10.2 and $1.1 \mathrm{eV}$, respectively. ${ }^{28}$

In this Letter, we determine absolute energy levels of liquid water comprising the EA, the IP, and the SHE level vs. vacuum. For this purpose, we first model the liquid surface and calculate the potential offset across the water-vacuum interface. The computational SHE level is found to lie at $4.56 \mathrm{eV}$ below the vacuum level, thus differing by only $0.11 \mathrm{eV}$ from the Trasatti reference $(4.44 \mathrm{eV}) .{ }^{20}$ For the EA and IP, we tune a hybrid functional to reproduce the band gap achieved with $\mathrm{QS} G \tilde{W}$ calculations, which corresponds to the most accurate method for determining band edges. The resulting picture is found to be consistent with both the measured range of IPs and the value of $V_{0}$ inferred from the hydrated electron.

To model the water-vacuum interface, we first carry out ab initio molecular dynamics (MD) simulations of liquid water with classical nuclei in the NVT ensemble. We use an orthorhombic periodic supercell ( $a=12.42$ and $c=24.48 \AA$ ) containing 384 water molecules at the experimental density of liquid water $\left(1 \mathrm{~g} / \mathrm{cm}^{3}\right)$. Then, we enlarge the simulation cell in the $c$ direction to accommodate $40 \AA$ of vacuum $(a=12.42$ and $c=64.48 \AA)$ and evolve the MD further for $60 \mathrm{ps}$ [cf. Supplementary Information (SI) ${ }^{29}$ ]. This computational setup has been shown to be sufficient for achieving proper potential offsets, when compared to simulations with larger supercells and with quantum nuclei. ${ }^{8}$ We use a rVV10 functional that has been designed to reproduce the experimental density of bulk liquid water. ${ }^{30}$ In the MD simulation of the slab, the averaged mass density of water in the bulk region amounts to $0.995 \mathrm{~g} / \mathrm{cm}^{3}$ [cf. Fig. 1(a)], thus differing from the target value by only $0.005 \mathrm{~g} / \mathrm{cm}^{3}$. Such a difference leads to negligible differences in the potential offset, as can be inferred from the calculated deformation potential of liquid water. ${ }^{31}$

During the MD simulation, we monitor electric field in the vacuum region finding oscilla- 
tions without any drift around an average value of only $0.004 \mathrm{eV} / \AA$ (cf. $\left.\mathrm{SI}^{29}\right)$ to be compared with the average value of $0.005 \mathrm{eV} / \AA$ achieved in Ref. 32 for a classical simulation lasting 1 nanosecond. In the calculation of $\Delta V$ In the calculation of the potential offset $\Delta V$ between the bulk region of the water slab and the vacuum level, the potential profile is symmetrized, thereby effectively eliminating the bias due to a residual electric field. Upon MD evolution, $\Delta V$ converges to a value of $3.69 \pm 0.04 \mathrm{eV}$, where the error is calculated performing a blocking analysis [cf. Fig. 1(b)]. ${ }^{33}$ This value appears to have converged, as time averages over the duration of 1 picosecond are found to oscillate around the average value, without showing any drifting behaviour. ${ }^{29}$ We also notice that recalculation of $\Delta V$ at the hybrid functional level ${ }^{29}$ produces an almost negligible difference $\left(0.06 \mathrm{eV}^{29}\right)$, in line with previous observations. ${ }^{34-36}$ The present value of $\Delta V$ is similar to other calculations in the literature (3.63-3.97 eV $),{ }^{8,32}$ but it should be reminded that it depends in principle on the functional and the pseudopotentials and can thus only be used for internal alignments within a given computational set-up.

To achieve a water-vacuum interface at which the water molecules are randomly oriented, we also construct water slabs from structural configurations obtained with simulations of bulk liquid water. To this aim, we take a cubic 64-water-molecule supercell, double it along one direction, cut it on both sides avoiding the formation of dangling bonds, and add a vacuum layer. This procedure allows us to produce slabs of the same size as those achieved in the MD simulation of the water-vacuum interface, but in which the water dipoles at the interface are oriented randomly. Considering a set of 2400 structures constructed in this way, we calculate a potential offset of $\Delta V_{\text {random }}=3.54 \mathrm{eV}$ for such an interface. The availability of $\Delta V$ and $\Delta V_{\text {random }}$ allows us to distinguish between quadrupolar and dipolar contributions to the potential offset. In particular, we find that $\Delta V$ is dominated by quadupolar contributions, which do not depend on the interface structure, as also observed in Ref. 32. The calculated dipolar contribution of $0.15 \mathrm{eV}$ is consistent with a previous theoretical study ${ }^{32}$ and agrees well with available estimates for the dipolar surface potential of water (0.14-0.17 eV, Refs. 
37 and 38).
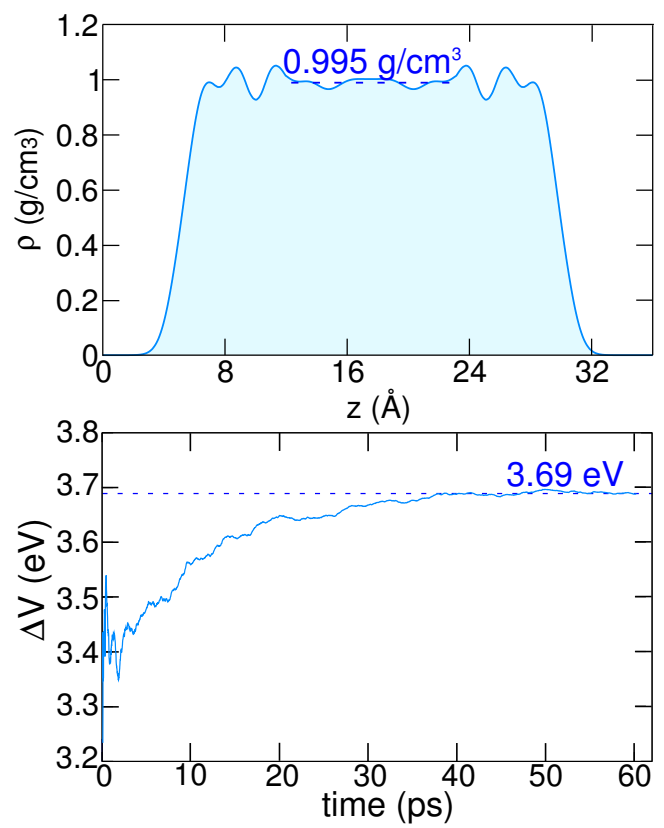

Figure 1: (a) Planar averaged mass density of liquid water at the interface with vacuum. (b) Cumulative running average of the potential offset $\Delta V$ across the water-vacuum interface. The dashed line indicates the converged value.

To achieve a physically meaningful reference level of bulk liquid water, we focus on the computational SHE level. ${ }^{15,19}$ This corresponds to the redox level $\mu_{\text {SHE }}$ associated to the reduction of the aqueous hydronium ion:

$$
\mathrm{H}_{3} \mathrm{O}^{+}(\mathrm{aq})+e^{-} \rightarrow \mathrm{H}_{2} \mathrm{O}(\ell)+\frac{1}{2} \mathrm{H}_{2}(\mathrm{~g}) .
$$

The level $\mu_{\mathrm{SHE}}$ can be aligned with respect to the average electrostatic potential of liquid water by using the grand-canonical formulation of solutes in aqueous solution and the thermodynamic integration method. ${ }^{19,29}$ Hence, by combining this result with the potential offset at the water-vacuum interface, we place $\mu_{\mathrm{SHE}}$ at $4.56 \mathrm{eV}$ below the vacuum level, very close to the value of $4.44 \mathrm{eV}$ proposed by Trasatti. ${ }^{20}$ This agreement therefore allows one to align in a reliable way the band structure of bulk water to the vacuum level. Since the $\mu_{\mathrm{SHE}}$ is a redox level, the calculated alignment is expected to be robust and to depend only very weakly on the adopted density functional, ${ }^{19,29}$ in perfect analogy with defect levels in 
semiconductors and insulators. ${ }^{39,40}$

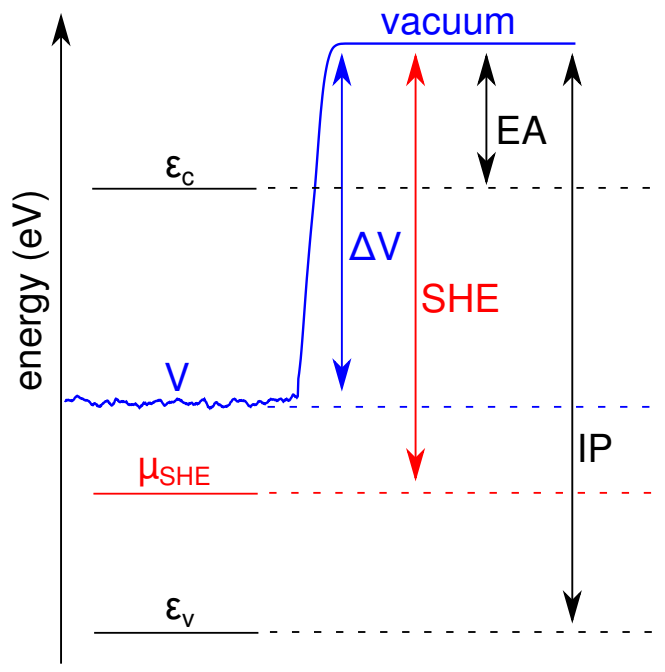

Figure 2: Schematic representation of the alignment of the standard hydrogen electrode level $\mu_{\text {SHE }}$ at the water-vacuum interface. We also report the alignment of the valence and conduction band edges $\epsilon_{\mathrm{v}}$ and $\epsilon_{\mathrm{c}}$, which define the ionization potential (IP) and electron affinity (EA), respectively.

Next, we focus on the IP and EA of liquid water. Taking advantage of the potential offset across the water-vacuum interface, we can now place the valence and conduction band edges $\left(\epsilon_{\mathrm{v}}\right.$ and $\left.\epsilon_{\mathrm{c}}\right)$ obtained in the QSGW$\tilde{W}$ scheme with respect to the vacuum level (cf. Fig. 2). This gives $\mathrm{IP}=9.5 \mathrm{eV}$ and $\mathrm{EA}=0.6 \mathrm{eV}$ (cf. Fig. 3). However, while the QSG $\tilde{W}$ scheme $^{6}$ is among the most accurate schemes for band gap calculations scoring a mean average error of $0.13 \mathrm{eV}$ on an extended set of materials, ${ }^{25-27}$ its performance in the determination of band edges is less impressive with errors up to $0.5 \mathrm{eV} .{ }^{16,25}$ Therefore, we turn to an alternative scheme, which consists in using the $\operatorname{PBE} 0(\alpha)$ hybrid functional ${ }^{41,42}$ with the fraction of Fock exchange $\alpha$ set to reproduce the correct band gap. This scheme has proved successful for the determination of band edges in a large variety of cases, including ionization potentials at surfaces, ${ }^{16,18,43}$ offsets at solid-solid ${ }^{34,35}$ and solid-liquid interfaces, ${ }^{16,18}$ and in-gap defect levels. ${ }^{44,45}$ To apply this scheme to liquid water, it is imperative to account for the quantum motion of the nuclei, which has been found to produce a sizable band gap renormalization. Hence, we use the $\operatorname{PBE} 0(\alpha)$ functional on top of configurations of liquid water achieved with path-integral ab initio MD based on the rVV10 functional. ${ }^{6}$ The band edges are extracted 
from a linear extrapolation of the density of states, as this procedure has been proved not be subject to tedious finite-size effects for both the valence ${ }^{19}$ and the conduction ${ }^{8,46}$ band edges. We retain $\alpha=45 \%$, for which the extracted band gap is found to coincide with the QSG $\tilde{W}$ band gap of $8.9 \mathrm{eV} .{ }^{6}$ Consequently, we obtain 9.7 and $0.8 \mathrm{eV}$ for the IP and the EA, respectively (cf. Fig. 3). These values do not depend on the adopted trajectories, as configurations from a path-integral simulation based on the SCAN $+\mathrm{rVV} 10$ functional $^{47}$ yield the same results within $0.03 \mathrm{eV}$.

We remark that both the valence and the conduction band-edge states, as probed by vertical extraction or injection of electrons, correspond to electronic states delocalized over the entire simulation cell, ${ }^{6,8}$ indicating that delocalization of these states iexceeds this length scale. More localized electronic states are only found when extra charges are considered. An extra hole results in the splitting of a water molecule and in the formation of the electrondeficient hydroxyl radical, ${ }^{48}$ while an extra electron develops in a hydrated electron characterized by a radius of $1.8 \AA .^{7}$

It is highly satisfactory that the present values are consistent with the current experimental characterization. Indeed, the calculated IP falls within the experimental range (9.3-10.1 $\mathrm{eV})^{9-12}$ while the calculated EA closely matches the value of $V_{0}=-0.97 \mathrm{eV}$ inferred in Ref. 7 from thermodynamical data for the hydrated electron. ${ }^{13,14}$ Early optical absorption spectra of liquid water feature an excitonic peak at 8.6-8.7 eV..$^{49,50}$ Considering that a value of $0.5 \mathrm{eV}$ has been recently estimated for the excitonic binding energy for liquid water ${ }^{51}$ this would imply a fundamental gap of 9.1-9.2 eV, close to our ab initio estimate. Nevertheless, more up to date measurements are required to further support these findings as this kind of experiments are subject to serious difficulties related to the presence of the water vapour at the interface. ${ }^{52}$

It is also of interest to compare our result with recent theoretical studies on the electronic structure of liquid water. In particular, Gaiduk et al. have recently proposed an alignment of energy levels through $\mathrm{G}_{0} \mathrm{~W}_{0}$ calculations based on dielectric-dependent hybrid functionals. ${ }^{8}$ 
The calculated band gap for bulk liquid water was found to be $10.05 \mathrm{eV}$, i.e. larger by $1.15 \mathrm{eV}$ than proposed in the present work. The alignment of the energy levels trough a water-vacuum slab resulted in values of the IP and the EA equal to -10.25 and $-0.2 \mathrm{eV}$, respectively (cf. Fig. 3). Their calculated IP falls slightly outside the defined experimental window. Moreover, the energy separation between the IP and the vertical ionization potential of the hydroxyde ion is $\sim 2 \mathrm{eV}$, larger by $0.5 \mathrm{eV}$ than the upper limit defined by the experiment, ${ }^{11}$ and larger by $1.3 \mathrm{eV}$ than a previous theoretical study. ${ }^{53}$ Such a stark difference may be motivated by the absence of vertex corrections in the $G W$ scheme employed in Ref. 8. To test this possibility, we also report in Fig. 3 the energy alignment achieved at the $\mathrm{QS} G W$ level without vertex corrections in Ref. 6. The QSGW gap is $9.8 \mathrm{eV}$, while $\mathrm{IP}=-10.0 \mathrm{eV}$ and $\mathrm{EA}=0.2 \mathrm{eV}$, all these values being close to those calculated in Ref. 8. Finally, it should be noted that if the value of EA of Ref. 8 is included in the thermodynamic model of Ref. 7, the resulting redox level of the hydrated electron would differ from the experimental characterization by as much as $0.6 \mathrm{eV}$.

In a recent $a b$ initio study based on $G W_{0}$ calculations, Ziae and Bredow have determined a band gap of $9.1 \mathrm{eV}$, and values for the IP and the EA at 10.2 and $1.1 \mathrm{eV}$, respectively. ${ }^{28}$ The band gap and the EA differ by only $0.2-0.3 \mathrm{eV}$ from those calculated in this work and the EA also agrees well with the thermodynamic model of Ref. 7. However, these authors have relied on the offset determined by Gaiduk et al. with a different computational set-up. ${ }^{8}$ Since the electrostatic potential in pseudopotential approaches does not correspond to an absolute physical quantity, such a combination of results leads to inconsistencies and may not be accurate.

In conclusion, we provided a detailed description of the absolute energy levels of liquid water by combining several state-of-the-art methodologies. After determining the potential offset at the water-liquid interface, we found the SHE level at $4.56 \mathrm{eV}$ below the vacuum level, in excellent agreement with the experimental reference value of $4.44 \mathrm{eV}$. Furthermore, the band edges determined in this work give an ionization potential of $9.7 \mathrm{eV}$ and an elec- 


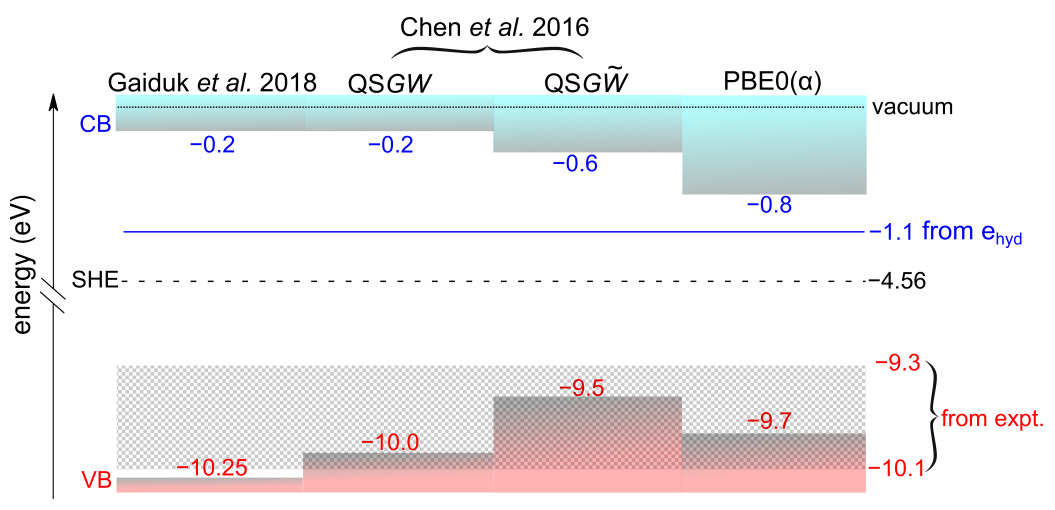

Figure 3: Band alignment of liquid water as achieved with the QSG $\tilde{W}$ scheme and with the $\operatorname{PBE0}(\alpha)$ functional, compared to experimental data for the IP (Refs. 9,10,12) and with the conduction band edge inferred from the hydrated electron $e_{\text {hyd }}$ (Ref. 7). Energies are given in $\mathrm{eV}$ and are referred to the vacuum level.

tron affinity of $0.8 \mathrm{eV}$, consistent with the available characterization from photoemission experiments, optical measurements, and thermodynamical data for the hydrated electron.

\section{Acknowledgement}

We thank Wei Chen, Nicolas Hörmann, and Julia Wiktor for fruitful discussions. This work has been performed in the context of the National Center of Competence in Research (NCCR): Materials' Revolution: Computational Design and Discovery of Novel Materials (MARVEL) of the Swiss National Science Foundation. We used computational resources of CSCS and SCITAS-EPFL.

\section{References}

(1) Ball, P. Water: Water - an Enduring Mystery. Nature 2008, 452, 291-292.

(2) Ball, P. Astrochemistry and Astrobiology; Springer-Verlag: Berlin Heidelberg, 2013; pp 169-210. 
(3) Turi, L.; Rossky, P. J. Theoretical Studies of Spectroscopy and Dynamics of Hydrated Electrons. Chem. Rev. 2012, 112, 5641-5674.

(4) Bernas, A.; Ferradini, C.; Jay-Gerin, J. On the Electronic Structure of Liquid Water: Facts and Reflections. Chem. Phys. 1997, 222, 151-160.

(5) Coe, J. V.; Earhart, A. D.; Cohen, M. H.; Hoffman, G. J.; Sarkas, H. W.; Bowen, K. H. Using Cluster Studies to Approach the Electronic Structure of Bulk Water: Reassessing the vacuum Level, Conduction Band Edge, and Band Gap of Water. J. Chem. Phys. 1997, 10\%, 6023-6031.

(6) Chen, W.; Ambrosio, F.; Miceli, G.; Pasquarello, A. Ab initio Electronic Structure of Liquid Water. Phys. Rev. Lett. 2016, 117, 186401.

(7) Ambrosio, F.; Miceli, G.; Pasquarello, A. Electronic Levels of Excess Electrons in Liquid Water. J. Phys. Chem. Lett. 2017, 8, 2055-2059.

(8) Gaiduk, A. P.; Pham, T. A.; Govoni, M.; Paesani, F.; Galli, G. Electron Affinity of Liquid Water. Nat. Comm. 2018, 9, 247.

(9) Winter, B.; Weber, R.; Widdra, W.; Dittmar, M.; Faubel, M.; Hertel, I. V. Full Valence Band Photoemission from Liquid Water Using EUV Synchrotron Radiation. J. Phys. Chem. A 2004, 108, 2625-2632.

(10) Winter, B.; Faubel, M.; Hertel, I. V.; Pettenkofer, C.; Bradforth, S. E.; JagodaCwiklik, B.; Cwiklik, L.; Jungwirth, P. Electronic Binding Energies of Hydrated $\mathrm{H}_{3} \mathrm{O}^{+}$ and $\mathrm{OH}^{-}$: Photoelectron Spectroscopy of Aqueous Acid and Base Solutions Combined with Electronic Structure Calculations. J. Am. Chem. Soc. 2006, 128, 3864-3865.

(11) Winter, B.; Faubel, M.; Vácha, R.; Jungwirth, P. Behavior of Hydroxide at the Water/Vapor Interface. Chem. Phys. Lett. 2009, 474, $241-247$. 
(12) Seidel, R.; Thürmer, S.; Winter, B. Photoelectron Spectroscopy Meets Aqueous Solution: Studies from a Vacuum Liquid Microjet. J. Phys. Chem. Lett. 2011, 2, 633-641.

(13) Schwarz, H. A. Enthalpy and Entropy of Formation of the Hydrated Electron. J. Phys. Chem. 1991, 95, 6697-6701.

(14) Jou, F.-Y.; Freeman, G. R. Temperature and Isotope Effects on the Shape of the Optical Absorption Spectrum of Solvated Electrons in Water. J. Phys. Chem. 1979, 83, 23832387.

(15) Cheng, J.; Sprik, M. Alignment of Electronic Energy Levels at Electrochemical Interfaces. Phys. Chem. Chem. Phys. 2012, 14, 11245-11267.

(16) Guo, Z.; Ambrosio, F.; Chen, W.; Gono, P.; Pasquarello, A. Alignment of Redox Levels at Semiconductor-Water Interfaces. Chem. Mater. 2018, 30, 94-111.

(17) Ambrosio, F.; Wiktor, J.; Pasquarello, A. pH-Dependent Surface Chemistry from FirstPrinciples: Application to the $\mathrm{BiVO}_{4}(010)$-Water Interface. ACS Appl. Mater. \& Interfaces 2018, 10.1021/acsami.7b16545.

(18) Ambrosio, F.; Wiktor, J.; Pasquarello, A. pH-Dependent Catalytic Reaction Pathway for Water Splitting at the $\mathrm{BiVO}_{4}-$ Water Interface from the Band Alignment. ACS Energy Lett. 2018, 3, 829-834.

(19) Ambrosio, F.; Miceli, G.; Pasquarello, A. Redox Levels in Aqueous Solution: Effect of van der Waals Interactions and Hybrid Functionals. J. Chem. Phys. 2015, 143, 244508.

(20) Trasatti, S. The Absolute Electrode Potential: an Explanatory Note (Recommendations 1986). Pure Appl. Chem. 1986, 58, 955-966.

(21) Adriaanse, C.; Cheng, J.; Chau, V.; Sulpizi, M.; VandeVondele, J.; Sprik, M. Aqueous Redox Chemistry and the Electronic Band Structure of Liquid Water. J. Phys. Chem. Lett. 2012, 3, 3411-3415. 
(22) Pham, T. A.; Zhang, C.; Schwegler, E.; Galli, G. Probing the Electronic Structure of Liquid Water with Many-Body Perturbation Theory. Phys. Rev. B 2014, 89, 060202.

(23) Del Ben, M.; Hutter, J.; VandeVondele, J. Probing the Structural and Dynamical Properties of Liquid Water with Models Including Non-Local Electron Correlation. J. Chem. Phys. 2015, 143, 054506.

(24) Shishkin, M.; Marsman, M.; Kresse, G. Accurate Quasiparticle Spectra from SelfConsistent $G W$ Calculations with Vertex Corrections. Phys. Rev. Lett. 2007, 99, 246403.

(25) Chen, W.; Pasquarello, A. Accurate Band Gaps of Extended Systems via Efficient Vertex Corrections in GW. Phys. Rev. B 2015, 92, 041115.

(26) Wiktor, J.; Reshetnyak, I.; Ambrosio, F.; Pasquarello, A. Comprehensive Modeling of the Band Gap and Absorption Spectrum of $\mathrm{BiVO}_{4}$. Phys. Rev. Materials 2017, 1, 022401.

(27) Wiktor, J.; Rothlisberger, U.; Pasquarello, A. Predictive Determination of Band Gaps of Inorganic Halide Perovskites. J. Phys. Chem. Lett. 2017, 8, 5507-5512, PMID: 29077408 .

(28) Ziaei, V.; Bredow, T. Probing ionization Potential, Electron Affinity and Self-Energy Effect on the Spectral Shape and Exciton Binding Energy of Quantum Liquid Water with Self-Consistent Many-Body Perturbation Theory and Bethe-Salpeter Equation. $J$. Phys.: Condens. Matter 2018,

(29) The Supplementary Information contains computational details on the molecular dynamics simulations, the time evolution of the electric field and the potential offset, a description of the computational SHE, and additional Refs. 54-69. 
(30) Miceli, G.; de Gironcoli, S.; Pasquarello, A. Isobaric First-Principles Molecular Dynamics of Liquid Water with Nonlocal van der Waals Interactions. J. Chem. Phys. 2015, 142, 034501.

(31) Wiktor, J.; Bruneval, F.; Pasquarello, A. Partial Molar Volumes of Aqua Ions from First Principles. J. Chem. Theory Comput. 2017, 13, 3427-3431, PMID: 28683201.

(32) Leung, K. Surface Potential at the AirWater Interface Computed Using Density Functional Theory. J. Phys. Chem. Lett. 2010, 1, 496-499.

(33) Flyvbjerg, H.; Petersen, H. G. Error Estimates on Averages of Correlated Data. J. Chem. Phys. 1989, 91, 461-466.

(34) Alkauskas, A.; Broqvist, P.; Devynck, F.; Pasquarello, A. Band Offsets at Semiconductor-Oxide Interfaces from Hybrid Density-Functional Calculations. Phys. Rev. Lett. 2008, 101, 106802.

(35) Steiner, K.; Chen, W.; Pasquarello, A. Band offsets of Lattice-Matched Semiconductor Heterojunctions Through Hybrid Functionals and $G_{0} W_{0}$. Phys. Rev. B 2014, 89, 205309.

(36) Shaltaf, R.; Rignanese, G.-M.; Gonze, X.; Giustino, F.; Pasquarello, A. Band Offsets at the $\mathrm{Si} / \mathrm{SiO}_{2}$ Interface from Many-Body Perturbation Theory. Phys. Rev. Lett. 2008, 100, 186401.

(37) Cheng, J.; Sprik, M. Alignment of Electronic Energy Levels at Electrochemical Interfaces. Phys. Chem. Chem. Phys. 2012, 14, 11245-11267.

(38) Isse, A. A.; Gennaro, A. Absolute Potential of the Standard Hydrogen Electrode and the Problem of Interconversion of Potentials in Different Solvents. J. Phys. Chem. B 2010, 114, 7894-7899. 
(39) Alkauskas, A.; Broqvist, P.; Pasquarello, A. Defect Energy Levels in Density Functional Calculations: Alignment and Band Gap Problem. Phys. Rev. Lett. 2008, 101, 046405.

(40) Alkauskas, A.; Broqvist, P.; Pasquarello, A. Defect levels Through Hybrid Density Functionals: Insights and applications. Phys. Status Solidi B 2011, 248, 775-789.

(41) Perdew, J. P.; Ernzerhof, M.; Burke, K. Rationale for Mixing Exact Exchange with Density Functional Approximations. J. Chem. Phys. 1996, 105, 9982-9985.

(42) Adamo, C.; Barone, V. Toward Reliable Density Functional Methods without Adjustable Parameters: The PBE0 Model. J. Chem. Phys. 1999, 110, 6158-6170.

(43) Chen, W.; Pasquarello, A. Band-edge positions in $G W$ : Effects of starting point and self-consistency. Phys. Rev. B 2014, 90, 165133.

(44) Komsa, H.-P.; Pasquarello, A. Assessing the Accuracy of Hybrid Functionals in the Determination of Defect Levels: Application to the As Antisite in GaAs. Phys. Rev. B 2011, 84, 075207 .

(45) Chen, W.; Pasquarello, A. Accuracy of $G W$ for Calculating Defect Energy Levels in Solids. Phys. Rev. B 2017, 96, 020101.

(46) Prendergast, D.; Grossman, J. C.; Galli, G. The Electronic Structure of Liquid Water within Density-Functional Theory. J. Chem. Phys. 2005, 123, 014501.

(47) Wiktor, J.; Ambrosio, F.; Pasquarello, A. Note: Assessment of the SCAN+rVV10 Functional for the Structure of Liquid Water. J. Chem. Phys. 2017, 147, 216101.

(48) Ma, J.; Schmidhammer, U.; Pernot, P.; Mostafavi, M. Reactivity of the Strongest Oxidizing Species in Aqueous Solutions: The Short-Lived Radical Cation H2OâĂćt. J. Phys. Chem. Lett. 2014, 5, 258-261.

(49) Heller, J.; Hamm, R.; Birkhoff, R.; Painter, L. Collective Oscillation in Liquid Water. J. Chem. Phys. 1974, 60, 3483-3486. 
(50) Shibaguchi, T.; Onuki, H.; Onaka, R. Electronic-Structures of Water and Ice. J. Phys. Soc. Jpn. 1977, 42, 152-158.

(51) Garbuio, V.; Cascella, M.; Kupchak, I.; Pulci, O.; Seitsonen, A. P. Proton Disorder in Cubic Ice: Effect on the Electronic and Optical Properties. J. Chem. Phys. 2015, 143, 084507 .

(52) Hayashi, H.; Watanabe, N.; Udagawa, Y.; Kao, C.-C. The complete optical spectrum of liquid water measured by inelastic x-ray scattering. Proc. Natl. Acad. Sci. U.S.A. 2000, 97, 6264-6266.

(53) Opalka, D.; Pham, T. A.; Sprik, M.; Galli, G. The Ionization Potential of Aqueous Hydroxide Computed using Many-Body Perturbation Theory. J. Chem. Phys. 2014, $141,034501$.

(54) VandeVondele, J.; Krack, M.; Mohamed, F.; Parrinello, M.; Chassaing, T.; Hutter, J. Quickstep: Fast and Accurate Density Functional Calculations using a Mixed Gaussian and Plane Waves Approach. Comput. Phys. Commun. 2005, 167, $103-128$.

(55) Goedecker, S.; Teter, M.; Hutter, J. Separable Dual-Space Gaussian Pseudopotentials. Phys. Rev. B 1996, 54, 1703-1710.

(56) Hartwigsen, C.; Goedecker, S.; Hutter, J. Relativistic Separable Dual-Space Gaussian Pseudopotentials from H to Rn. Phys. Rev. B 1998, 58, 3641-3662.

(57) Dunning, T. H. Gaussian basis sets for Use in Correlated Molecular Calculations. I. The Atoms Boron Through Neon and Hydrogen. J. Chem. Phys. 1989, 90, 1007-1023.

(58) Vydrov, O. A.; Van Voorhis, T. Nonlocal van der Waals Density Functional: The Simpler the Better. J. Chem. Phys. 2010, 133, 244103.

(59) Sabatini, R.; Gorni, T.; de Gironcoli, S. Nonlocal van der Waals Density Functional Made Simple and Efficient. Phys. Rev. B 2013, 87, 041108. 
(60) Nosé, S. A Unified Formulation of the Constant Temperature Molecular Dynamics Methods. J. Chem. Phys. 1984, 81, 511-519.

(61) Hoover, W. G. Canonical Dynamics: Equilibrium Phase-Space Distributions. Phys. Rev. A 1985, 31, 1695-1697.

(62) Guidon, M.; Schiffmann, F.; Hutter, J.; VandeVondele, J. Ab Initio Molecular Dynamics Using Hybrid Density Functionals. J. Chem. Phys. 2008, 128, 214104.

(63) Guidon, M.; Hutter, J.; VandeVondele, J. Robust Periodic Hartree-Fock Exchange for Large-Scale Simulations Using Gaussian Basis Sets. J. Chem. Theory Comput. 2009, 5, 3010-3021.

(64) Guidon, M.; Hutter, J.; VandeVondele, J. Auxiliary Density Matrix Methods for Hartree-Fock Exchange Calculations. J. Chem. Theory Comput. 2010, 6, 2348-2364.

(65) Komsa, H.-P.; Rantala, T. T.; Pasquarello, A. Finite-Size Supercell Correction Schemes for Charged Defect Calculations. Phys. Rev. B 2012, 86, 045112.

(66) Freysoldt, C.; Grabowski, B.; Hickel, T.; Neugebauer, J.; Kresse, G.; Janotti, A.; Van de Walle, C. G. First-Principles Calculations for Point Defects in Solids. Rev. Mod. Phys. 2014, 86, 253-305.

(67) Ambrosio, F.; Miceli, G.; Pasquarello, A. Structrural, Dynamical and Electronic Properties of Liquid Water: a Hybrid Functional Study. J. Chem. Phys. B 2016, 120, 7456-7470.

(68) Kim, J.; Schmitt, U. W.; Gruetzmacher, J. A.; Voth, G. A.; Scherer, N. E. The Vibrational Spectrum of the Hydrated Proton: Comparison of Experiment, Simulation, and Normal Mode Analysis. J. Chem. Phys. 2002, 116, 737-746.

(69) Kelly, C. P.; Cramer, C. J.; Truhlar, D. G. Aqueous Solvation Free Energies of Ions 
and IonWater Clusters Based on an Accurate Value for the Absolute Aqueous Solvation Free Energy of the Proton. J. Phys. Chem. B 2006, 110, 16066-16081. 
Graphical TOC Entry

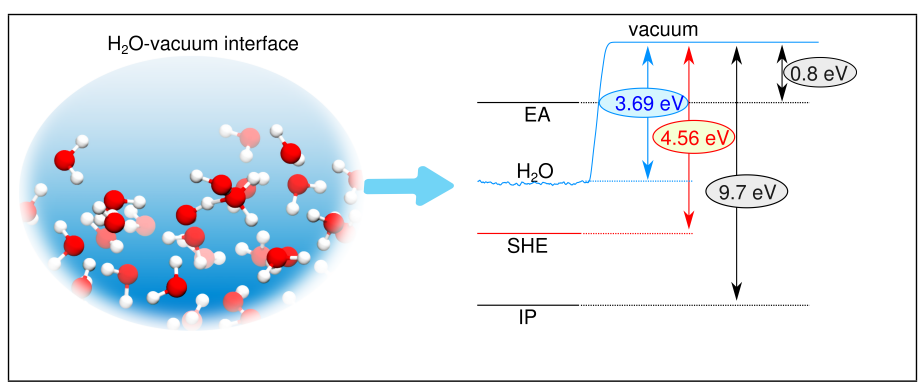

